Conference Proceedings Paper

\title{
Efficient SMA Actuation - Design \& Control Concepts
}

\author{
Paul Motzki * \\ Center for Mechatronics and Automa tion Technology (ZeMA) gGmbH, Sa arbruecken, Germany \\ * Corre spondence: p.motzki@zema.de; Tel.: +49-681-85787-545
}

\begin{abstract}
The versatility in form factors of thermal shape memory alloys (SMA) in combination with their unique actuation and sensing abilities allow for the design and construction of innovative multifunctional systems. Despite the considerable number of advantages, like their exceptional energy density, only a few SMA-based actuator systems are commercially available. One of the main reasons for this is their inefficient thermal activation and the resulting high energy consumption. The efficiency of SMA-based actuator systems can be improved by innovative design and control approaches. In the first part, the intelligent combination of SMA actuator wires with bi-stable, nonlinear spring elements is described. This combination eliminates oftentimes-quoted disadvantages of SMAs - slow actuation and energy-inefficiency - for a wide range of applications. In particular, tw o energy -free actuator configurations are realized, which can be applied to any nonproportional actuation tasks. The second approach for the realization of high-speed actuation and energy-efficiency is the activation of SMA wires with high voltage pulses, which leads to actuation times in the millisecond-range and energy-savings up to $80 \%$ in comparison to the suppliers' recommendations. It is show $\mathrm{n}$ that even high AC voltages like ty pical mains supplies can be directly used for highly efficient SMA activation.
\end{abstract}

Keywords: shape memory alloys;SMA; actuator; energy; efficiency; high voltage; bistable

\section{Introduction}

Shape Memory Alloy (SMA) actuators like the commonly used SMA wires are already present in a few commercially available products. The company Actuator Solutions $\mathrm{GmbH}$, for example, offers a variety of different gas and fluid valves as well as camera systems based on SMA wire actuators [1]. SMA actuator wires will contract when heated, in technical applications typically via an applied current. The contraction is a consequence of a phase transformation (Figure 1) between Martensite (cooled wire) and Austenite (heated wire) [2], [3]. In order to return to the extended length, the wires are coupled with an additional biasing mechanism (Figure 2), such as the gravitational force of a mass (a), a restoring coil spring (b), or the pulling force of a second SMA actuator (c) [4]. One drawback of an SMA actuator wire system, such as when coupled with a constant force or a coil spring, is the continuous energy needed to remain in the activated position [5]-[8]. The bias force keeps the not-activated SMA wire in a defined initial position. Activation and contraction of the SMA wire causes the SMA actuator to reach a second position. To remain in this second position, the SMA wirehas to be constantly heated, resulting in a continuous energy consumption. Also, a position or power control is necessary to keep the actuator in that defined second position, independently of environmental influences like temperature changes or increased convective cooling through higher air flow rates [9], [10]. The actuation stroke of the SMA wire is dependent on the its length (typically around $4 \% \max [11],[12])$. To produce large strokes, SMA actuators often need large construction 
space. The pulling force, as well as actuation cycling speed, is directly dictated by the diameter of the SMA wires [13], [14].
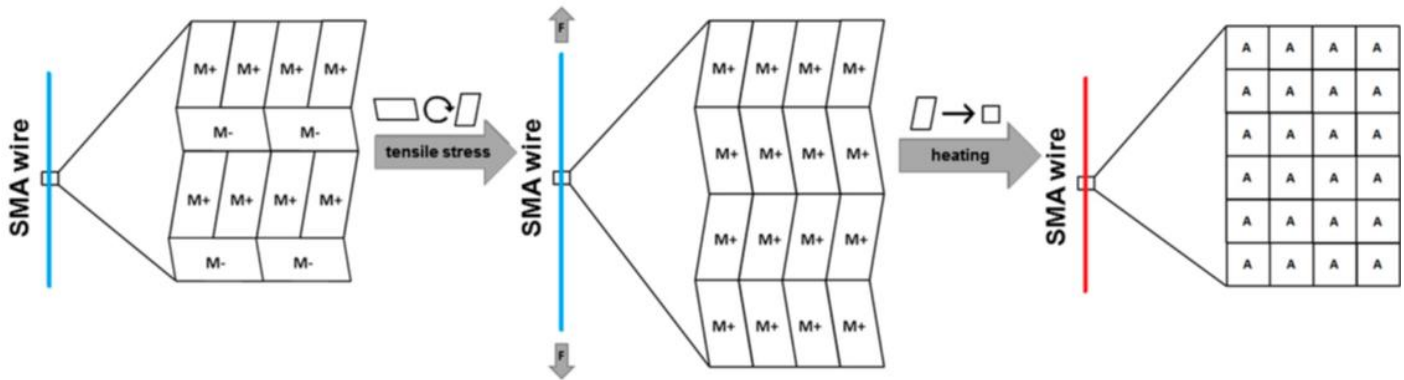

Figure 1. Schematic of martensitic and austenitic phase transformation in an SMA wire [15].

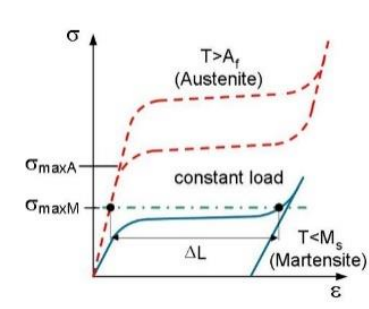

(a)
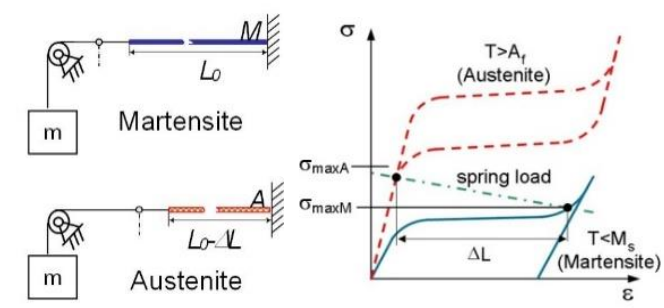

$\vec{\varepsilon}$

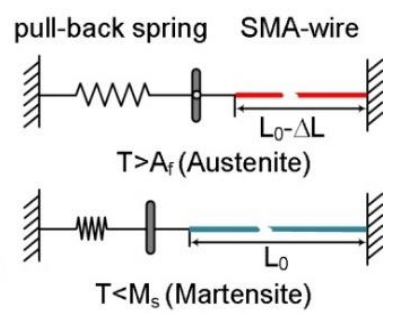

(b)

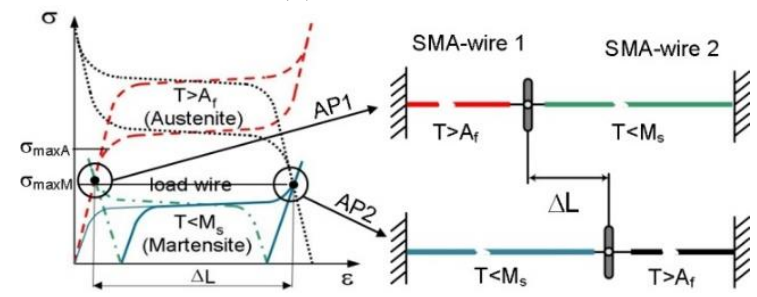

(c)

Figure 2. SMA wire actua tors with different biasing mechanisms: (a) constant mass, (b) linear spring, (c) antagonistic SMA wire [4].

To address these drawbacks mentioned above, a new SMA actuator design is presented. In this actuator, a bi-stable snapping mechanism is combined with an antagonistic SMA wire configuration. In this way, the actuator has two defined stable and energy-free positions. The SMA wires are only activated to switch between these two positions. The force output and the stroke of this actuator are only dependent on the snap design, rather than being directly linked to the SMA wires' lengths and diameters. This allows for the construction and the design of very compact and energy-efficient actuators. Additionally, the actuation frequencies are increased, because the antagonistic configuration provides active actuation in both directions, unlike in a SMA-spring combination.

Another way to improve energy-efficiency of SMA actuators is through the electrical control concepts. In current state-of-the-art actuators, the SMA wire activation takes place under nonadiabatic conditions, i.e., a large amount of the heating energy is lost due to heat exchange with the environment during the activation process. With high electrical power and a fast heating pulse, it is possible to reach the transformation temperature and start actuation before heat is lost to the environment, thus leading to an adiabatic activation [16]. This control concept can also be used for typical mains AC voltages and leads to energy-savings up to $80 \%$ in comparison to the traditional quasi-static activation of SMA wire actuators [17].

Following this introduction, the design of the bi-stableSMA actuation mechanism is presented in the first main chapter. The advantages of this innovative actuator design are illustrated at the example of the application of an industrial vacuum gripping system. The subsequent second main chapter introduces energy-efficient high voltage activation concepts. Systematic experimental studies are used to quantify energy-savings. The paper is concluded by a short summary of the presented results. 


\section{Bi-stable SMA Actuator Design Concept}

The core item in this energy-efficient actuator design is the bi-stable element. For the first studies, a thin beam out of sheet metal is used, but the actuator is not limited to this material or geometry [18]. The key to this new actuator design is the pivot-mounting of the bi-stable element. Figure 3 show s a basic combination of the bi-stable element and SMA wires and explains the actuator function principle.
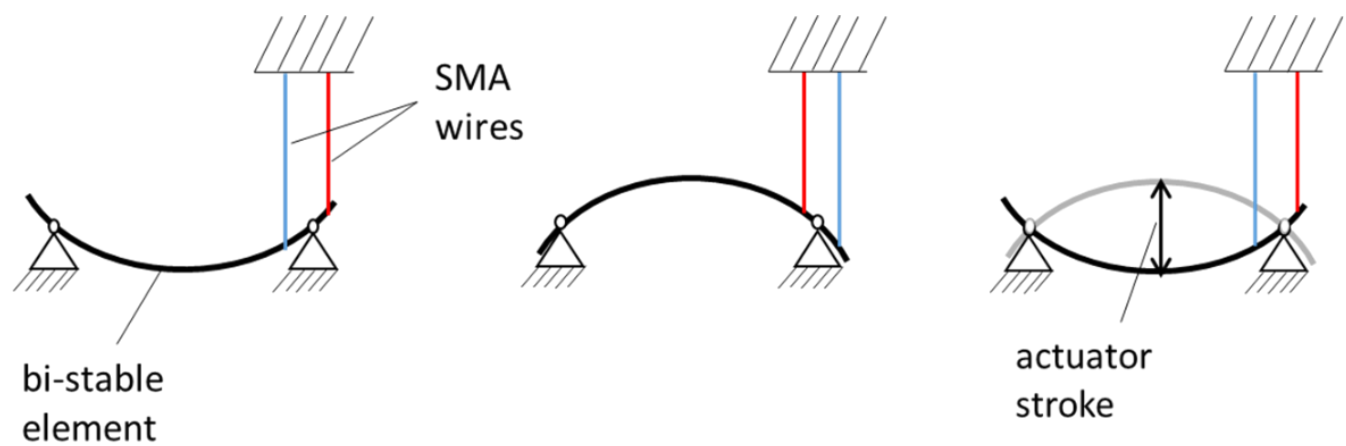

Figure 3. Functional principle of the bi-stable SMA actua tor [18].

In this example, the bi-stable element is mounted with two pivot joints. On each side of one of the joints, an SMA wire is attached. In this antagonistic configuration, the bi-stable element snaps into its second position whenever one of the SMA wires is activated. The maximum actuat ion stroke is observed in the center of the beam and depends on its pre-bending. The minimum length of the SMA wires depends on their attachment point. The closer they are attached to the pivot point, the less SMA wire stroke is needed to induce a snapping of the beam. At the same time, moreSMA force will be needed for closer attachment points, but SMA force can easily be scaled with a bigger wire diameter or the addition of more wires in a parallel mechanical configuration.

Using the bi-stable mechanism as a transmission element to convert the high energy density of the SMA wires into large strokes, the design space of the actuator can be reduced. The bistable actuator mechanism enables the design of high performance actuation system s with high stroke, high activation frequency, small design space and high energy efficiency [19].

As an exemplary implementation of this actuator, the realization of an industrial vacuum gripper is presented. A schematic view of the concept of the bistable SMA vacuum suction cup is show $n$ in Figure 4 [20]. The actuator mechanism again consists of a flexible deformable membrane, which is connected to a bistable spring. To actively switch between the two states, two antagonistic SMA wires are used. The orthogonal orientation of the levers related to the spring allows the parallel arrangement of the SMA wires to minimize the construction space. Activation of the bottom SMA wire lets the bistable mechanism switch from the lower to the upper position. After the position toggle of the bistable element the energy is switched off and the SMA wire cools down. When the membrane is sealed by the gripped object and deflected by the bistable element, a vacuum is generated inside the cavity between the membrane and the work piece. Release of the payload requires the activation of the top SMA wire. The energy-free holding of the two positions leads to an energy efficient, cycle time reduced and fail-safe actuation mechanism.

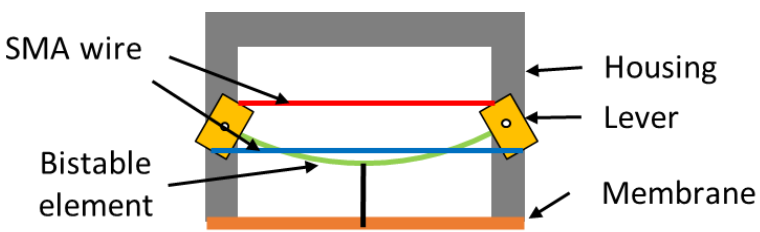


1. Standby

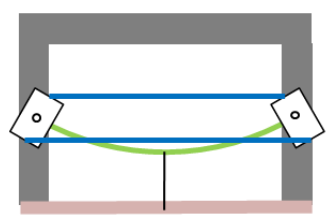

- No wire actuated

- Membrane in plane state

- Suction cup ready to grip
2. Gripping

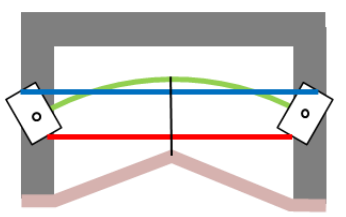

- Lower wire actuated

- Bi-stable spring snaps upwards

- Vacuum generation

- Gripping of workpiece
3. Holding

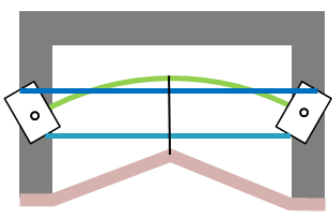

- No wire actuated

- Bi-stable spring maintains vacuum

- Workpiece is fixed

\section{Release}

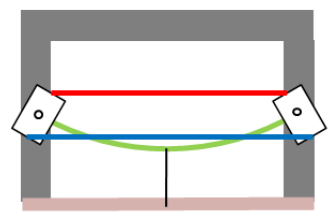

- Upper wire actuated

- Bi-stable spring snaps downwards

- Vacuum purge

- Workpiece released

Figure 4. Schematic view of the actuation concept from the bistable SMA vacuum suction cup [20].

The design space is minimized by fitting the geometry of the actuation mechanism to the rotational geometry of the membrane. The upper side of the mechanical framew ork of the actuation mechanism is presented in Figure 5. The membrane is directly connected to the bistable spring element. Maximizing the deformation force by a given ar ea and minimizing the manufacturing effort, the leaf spring is designed in a crosswise configuration with four clamping points. The ends of the leaf spring are attached to a clamping mechanism which is pivot-mounted to enable the state toggle (up and down). The clamping mechanism is symmetrically mounted in the ring with a rotational joint. Additionally, the clamp provides the mounting point for the lever of the upper and low er SMA wire. The lever is needed for the transformation of the linear SMA force in a rotary movement to toggle the state of the bistable element. For reducing the cooling time and using the design space efficiently the SMA element consists of bundles of thinner SAM wires (200 $\mu$ m diameter) to optimize the surface to volume ratio for faster convective cooling. The ends of the SMA elements are attached to the connection bar, which at the same time represents the port to the electronics. The actuator mechanism and the membrane as well as the electronics are integrated in a closed housing to meet the requirements for harsh industrial environments as shown in Figure 5. The presented new prototype of an innovativeSMA actuated bi-stable vacuum gripper enables an energy-efficient, failsafe, noiseless gripping system without the need for compressed air.

\section{High Voltage SMA Activation}

Commonly in applications, an electric current is used to heat the SMA wire through Joule heating. Usually, to control the SMA contraction, a current between zero and five Amperes (depending on the wire diameter) is recommended by the wire manufacturers [11], [12]. Therefore, the supply voltage must be adjusted according to the SMA wire's electrical resistance. Controlling SMA wires with this recommended values, however, results in slow actuation response due to the large time required to heat up the material and a lot of heat is lost to the environment, which results in low efficiency. As an alternative approach, short high voltage pulses can be used to achieve a fast heating of the SMA wire [16]. By means of this principle, it has been shown that it is possible to activate SMA wires much faster and in a more energy-efficient way. Even typical mains voltages, which supply alternating currents (AC) instead of direct current (DC), can be used for the activation of SMA wires und this control concept. The contraction can be controlled by varying the electric energy supplied by each pulse. For this purpose, the energy given to the wire is calculated in real time [17]. 

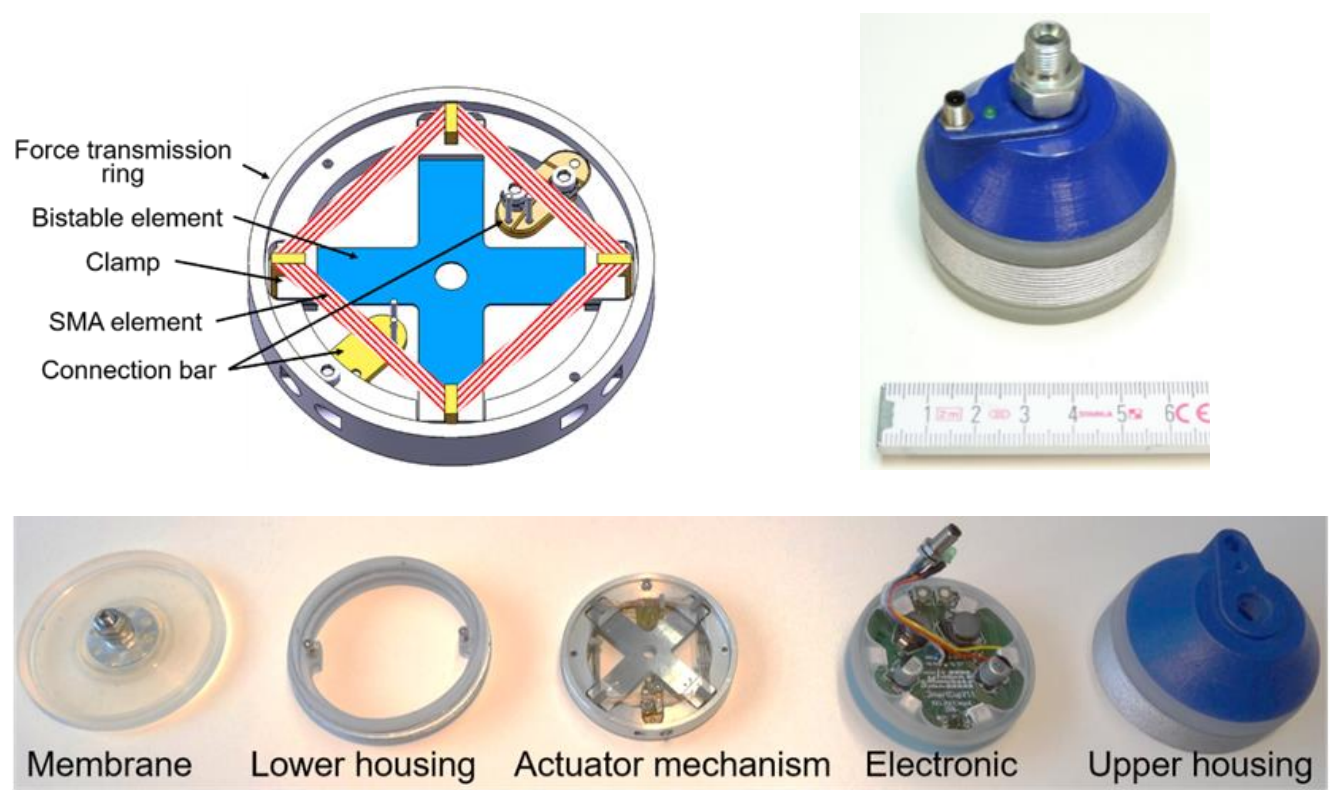

Figure 5. Mechanical actuation framework and photograph of the manufactured components for the bistable SMA vacuum suction cup [21]

The experimental setup is shown in Figure 6. A power supply is used to heat the SMA wire with an electric pulse. A laser displacement sensor measures the SMA wire stroke. The time derivatives of the displacement signal, i.e., velocity and acceleration, are calculated and plotted in post-processing. SMA force is recorded by a load cell of the type Futek LSB200. The voltage on the SMA wire is measured via 4-wires sensing, while electrical current measurement is realized via a current clamp. Additionally, the whole experiment is recorded with an optical high-speed camera system Olympus i-SPEED TR for a better interpretation of the measurement results. Signal processing and data acquisition during the experiment are realized via an NI CompactRIO system. A compression spring can be placed between the bottom clamp and a micro-adjustment stage, allowing to vary the SMA wire pre-tension. The air bearing guides the vertical motion with minimal friction. The laser displacement sensor detects the motion of the air bearing shaft. The SMA wire length in its austenitic state in this setup is $225 \mathrm{~mm}$.

In an exemplary experiment with DC supply, a $76 \mu \mathrm{m}$ SMA wire is activated in the conventional way using the suggested current of $150 \mathrm{~mA}$ in the datasheet [12]. The SMA wire is activated with an activation pulse width of $1 \mathrm{~s}$. The displacement reaches the value of $8.33 \mathrm{~mm}$, which corresponds to a $3.7 \%$ stroke. The same wire is then activated at increasing voltages. At each set voltage, the activation pulse time (pulse width) is slowly increased until a displacement of $8.33 \mathrm{~mm}$ is reached. In all experiments, the initial force in the SMA wire is $0.28 \mathrm{~N}$ and a spring rate of $0.056 \mathrm{~N} / \mathrm{mm}$ is used. The results of these experiments are shown in Table1. The activation delay describes the time interval from the start of activation until the bottom clamp of the SMA wire reaches maximum displacement of $8.33 \mathrm{~mm}$. The results show energy savings of almost $80 \%$ in comparison to the suggested quasistatic activation.

The same measurement sequence is performed for the SMA wire diameters of $50 \mu \mathrm{m}$ and $100 \mu \mathrm{m}$. The total energy consumption at the different supply voltages is illustrated in Figure 7. At low voltages, the thin wires need more energy to reach the same stroke. Thin wires have a higher surface-to-volume ratio and thus lose more energy to the environment during non-adiabatic activation. Between $12 \mathrm{~V}$ and $16 \mathrm{~V}$ the SMA wires start to reach the adiabatic region and the energy consumption settles at near constant values. At these voltages, the thin wires need the least amount of energy. In small diameter SMA wires is less material to be heated up before the actual phase transformation can begin. 


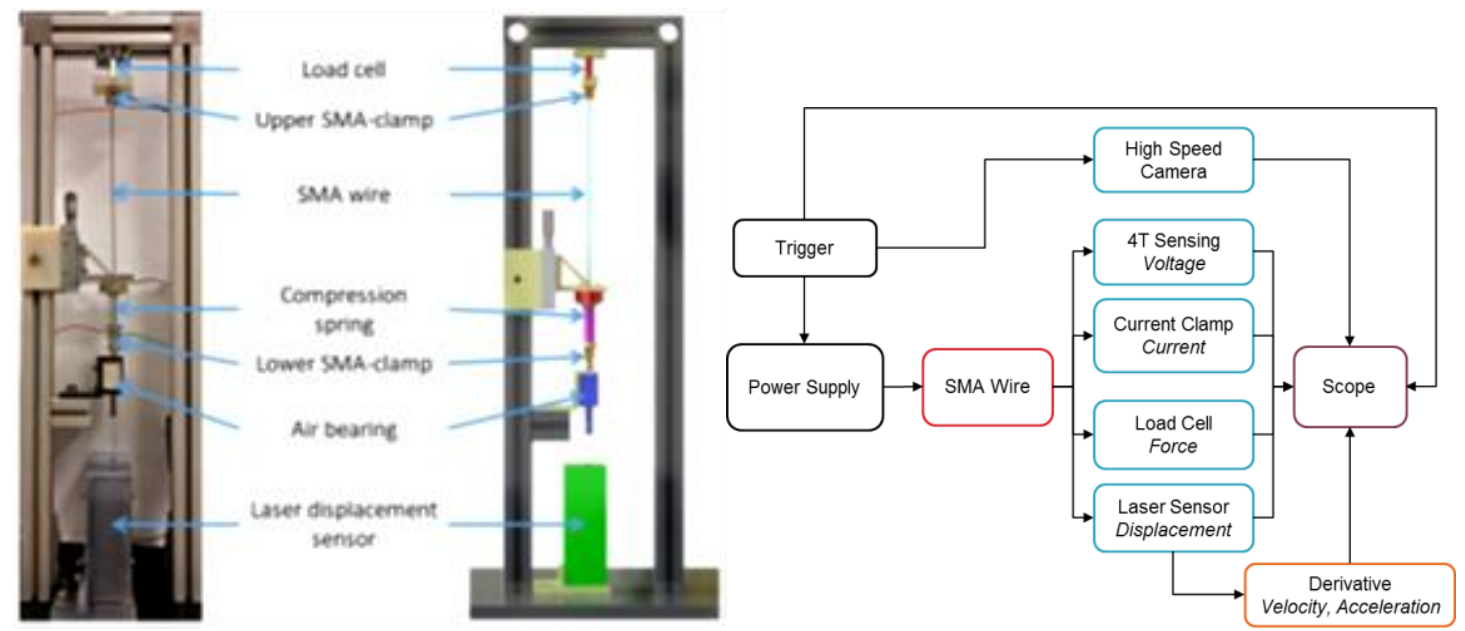

Figure 6. Mechanical structure and block diagram of the experimental setup [17], [22].

Table 1. Comparison of activation speed and energy consumption at different activa tion pulse widths for a $76 \mu \mathrm{m}$ SMA wire [22].

\begin{tabular}{cccccc}
\hline $\begin{array}{c}\text { Voltage } \\
{[\mathrm{V}]}\end{array}$ & $\begin{array}{c}\text { Displacement } \\
{[\mathrm{mm}]}\end{array}$ & $\begin{array}{c}\text { Pulse width } \\
{[\mathrm{ms}]}\end{array}$ & $\begin{array}{c}\text { Activation delay } \\
{[\mathrm{ms}]}\end{array}$ & $\begin{array}{c}\text { Energy } \\
{[\mathrm{J}]}\end{array}$ & $\begin{array}{c}\text { Ratio } \\
{[\%]}\end{array}$ \\
\hline 8 & 8.33 & 1000 & 1008 & 1.2 & 100 \\
12 & 8.33 & 226 & 237.1 & 0.637 & 53.1 \\
16 & 8.33 & 77 & 95.2 & 0.370 & 30.8 \\
24 & 8.33 & 29.2 & 57.2 & 0.305 & 25.4 \\
30 & 8.33 & 17.7 & 49 & 0.288 & 24 \\
35 & 8.33 & 12.8 & 45.8 & 0.282 & 23.5 \\
40 & 8.33 & 9.74 & 43.9 & 0.280 & 23.3 \\
48 & 8.33 & 6.71 & 42 & 0.277 & 23.1 \\
70 & 8.33 & 3.08 & 39.7 & 0.272 & 22.7 \\
110 & 8.33 & 1.21 & 38.5 & 0.267 & 22.3 \\
125 & 8.33 & 0.94 & 38.3 & 0.266 & 22.2 \\
\hline
\end{tabular}

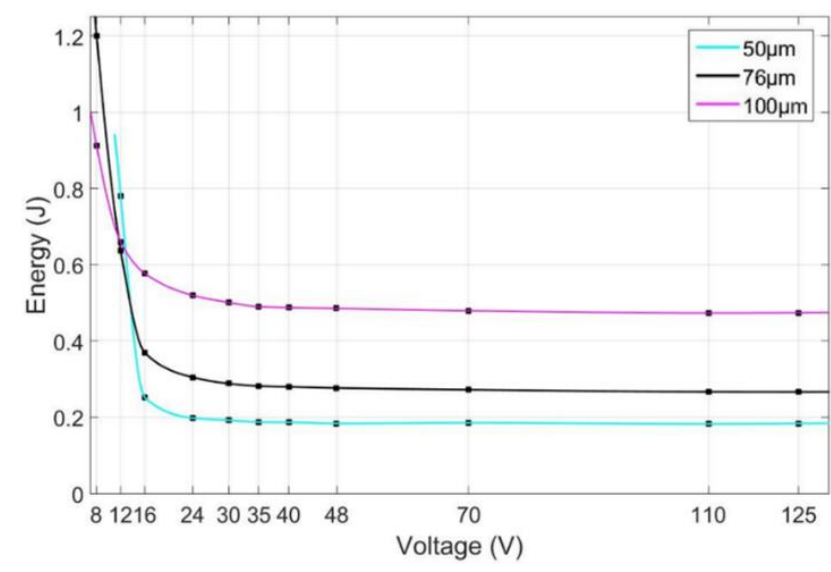

Figure 7. Total energy consumption for three diffe rent SMA wire diameters [22].

In the case of AC supply voltages, relatively high voltage levels are present. The mains voltage extends from $100 \mathrm{~V}_{\text {eff }}$ up to $240 \mathrm{~V}_{\text {eff }}$. Board voltage in ships can even be over $400 \mathrm{~V}$ eff. The high peak voltages in these AC supplies require to split the resulting current into pulses of short duration to 
prevent damage of the SMA wires. Therefore, a circuit board is developed to realize the pulse control of SMA actuators with AC voltage supplies. The concept of this control procedure is shown schematically in Figure 8. To simplify the circuit board and the necessary electronic components, the AC voltage first is rectified. After that, a MOSFET is used to switch the current through the wire on and off at any desired time.

Fluctuations in mains AC voltage supplies are common and often, fluctuations up to $10 \%$ are allowed. These deviations can have a negative impact on time-based pulses (like PWM control). Due to the high voltage levels in AC supplies, even low percentage deviations can affect the SMA wire's energy consumption in a non-negligible manner. To compensate such fluctuation and other noise in the voltage supply, a real-time energy measurement has been implemented. To calculate the converted energy in the SMA actuator, the voltage drops and the current through the wire are measured with a sample interval of $5 \mu$ s. With these measured values, every sampleinterval's energy is calculated and summed:

$$
W_{e l}=\sum_{i=1}^{n} U_{i} \cdot I_{i} \cdot \Delta t
$$

This real-time energy measurement allows to predefine an energy threshold for every pulse rather than a time duration. Thereby every single pulselength is nolonger predetermined. It depends on the actual voltage and thus on the actual current through the SMA wire.

The reference experiment for the AC measurements result from a conventional DC activation, where again the SMA actuator is activated with a DC voltage based on the prescribed current in the data sheet of the manufacturer (Figure 8, left-hand side). This results in a maximum displacement of $9.4 \mathrm{~mm}$. The number of pulses used in this systematic set of experiments varies from 10 to 100 . For every number of pulses, the energy threshold is gradually increased until the reference displacement is reached. A plot of the measurement with 100 pulses is shown in Figure 8 (right-hand side). The necessary overall energy equals that one used in the conventional DC activation (1200 mJ). Also, the flat displacement trajectory is similar to the conventional one.

If the number of active pulses is reduced to 10, the overall needed energy to reach the reference displacement is reduced to $440 \mathrm{~mJ}$, how ever the energy per every single pulse $(44 \mathrm{~mJ})$ is much higher (Figure 9). The reduced energy input can be explained with a faster activation and thus a lower energy loss to the environment during the heating of the SMA wire. With 10 pulses only $36.6 \%$ of energy is needed to reach the reference displacement.
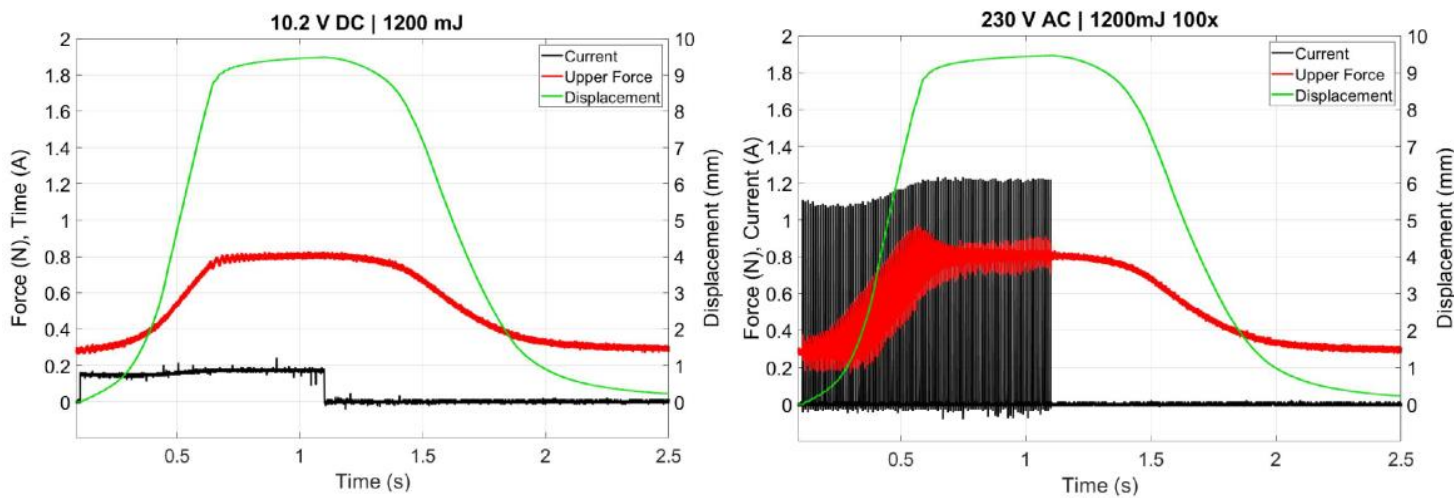

Figure 8. Mea surements of a common DC Activa tion (left-hand side) and pulsed AC activation (righthand side) with identicalenergy inputs [17]. 


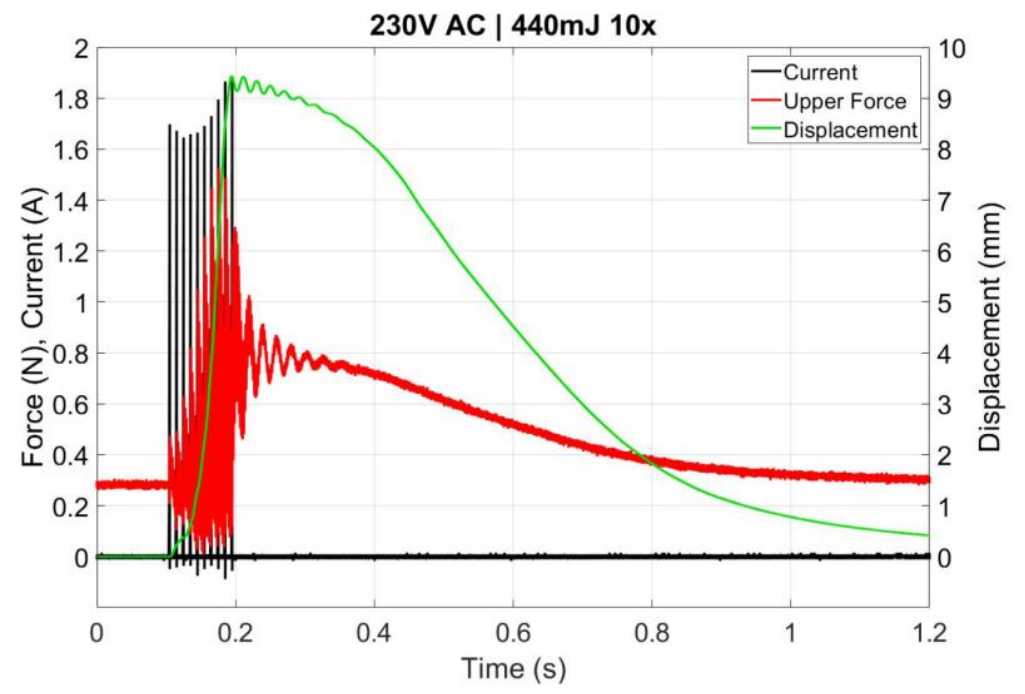

Figure 9. Measurements of a pulsed AC activa tion with ten high power pulses [17].

\section{Conclusion and Perspective}

This paper has given an overview of different methods to increase the low energy -efficiency of SMA actuator systems, which is well-known disadvantage of this technology. For a wide range of possible applications, a bi-stable design concept can lead to energy-free actuator positions. This mechanism has been illustrated at the example of an industrial vacuum gripper system. In addition to the mechanical design, efficiency can be increased by the demonstrated control concept of high voltage activation pulses. Experimental studies for DC and AC power supplies have shown possible energy-savings of $60-80 \%$ compared to conventional SMA control.

Future work will address the transfer of these concepts into different application-based prototypes and their validation. Especially the realization of compact power electronics for the control concept, which can be integrated in commercial systems, is one of the upcoming challenges.

Acknowledgments: This research received no external funding.

Conflicts of Interest: The authors declare no conflict of interest.

\section{Abbreviations}

The following abbreviations are used in this manuscript:

SMA: Shape memory alloy

AC: Alternating current

DC: Direct current

PWM: Pulse width modulation

MOSFET: Metaloxide semiconductor field-effect transistor

\section{References}

[1] Actuator Solutions GmbH and A. S. GmbH, "Actuator Solutions SMA Products," 2018. http://www.actua torsolutions.de/products/ (accessed Nov. 15, 2020).

[2] H. Janocha, Adaptronics and Smart Structures, 2. Berlin Heidelberg: SpringerVerlag, 2007.

[3] Y. Furuya and H. Shimada, “Shape memory actuators for robotic applications,” Mater. Des., 1991, doi: 10.1016/0261-3069(91)90088-L.

[4] VDE/VDI-Gesellschaft Mikoelektronik Mikro- und Feinwerktechnik (GMM), “Innovative Kleinantriebe," 1996. 
[5] J.M. Hollerbach, I. W. Hunter, and J. Ballantyne, “A compara tive analysis of actuator technologies for robotics," in The Robotics Review2, Cambridge: MIT Press, 1992, pp. 299-342.

[6] P. Motzki, F. Khelfa, L. Zimmer, M. Schmidt, and S. Seelecke, “Design and Valida tion of a Reconfigurable Robotic End-effector Based on Shape Memory Alloys," IEEE/ASME Trans. Mechatronics, vol. 24, no. 1, pp. 293-303, 2019, doi: 10.1109/TMECH.2019.2891348.

[7] P. Motzki and S. Seelecke, "Industrial Applications for Shape Memory Alloys," in Reference Module in Materials Science and Materials Engineering, Elsevier, 2019.

[8] O. Benafan et al., "Sha pe memory alloy actuator design: CASMART collaborative best practices and case studies," Int. J. Mech. Mater. Des., vol. 10, no. 1, pp. 1-42, 2014, doi: 10.1007/s10999-013-9227-9.

[9] S. J. Furst and S. Seelecke, "Modeling and experimental characterization of the stress, strain, and resistance of shape memory alloy actuator wires with controlled power input," J. Intell. Mater. Syst. Struct., vol. 23, no.11, pp. 1233-1247, May 2012, doi: 10.1177/1045389X12445036.

[10] N. Lewis, A. York, and S. Seelecke, “Experimental cha racterization of self-sensing SMA actuators under controlle d convective cooling," Smart Mater. Struct., vol. 22, no. 9, pp. 094012--, 2013, doi: 10.1088/0964$1726 / 22 / 9 / 094012$.

[11] SAES Getters, “SmartFlex Brochure,” 2017. https://www.saesgetters.com/sites/default/files/SmartFlex Brochure_2.pdf(accessed Nov. 15, 2020).

[12] Dynalloy Inc., “Technical Characteristics of Flexinol Actuator Wires," 2017. http://www.dynalloy.com/pdfs/TCF1140.pdf (accessed Nov.15, 2020).

[13] S. J.Furst, J.H. Crews, and S. Seelecke, “Characterization and modeling of opposing SMA-WIRE system for multifunctional, resistance-based controls applications," in ASME 2011 Conference on Smart Materials, Adaptive Structures and Intelligent Systems, SMASIS 2011, 2011, pp. 97-106, doi: https://doi.org/10.1115/SMASIS2011-4987.

[14] J. Luntz, B. Barnes, D. Brei, P. W. Alexander, A. Browne, and N. L. Johnson, “SMA wire actua tor modular design framework," 2009, doi: 10.1117/12.816752.

[15] R. Britz, P. Motzki, and S. Seelecke, "Scalable Bi-DirectionalSMA-Based Rotational Actua tor," Actuators, vol. 8, no.3, p. 60, Aug. 2019, doi: 10.3390/act8030060.

[16] P. Motzki, T. Gorges, M. Kappel, M. Schmidt, G. Rizzello, and S. Seelecke, "High-Speed and HighEfficiency Shape Memory Alloy Actuation," Smart Mater. Struct., Jun. 2018, doi: 10.1088/1361$665 X /$ aac9e1.

[17] T. Gorges, G. Rizzello, S. Seelecke, and P. Motzki, “High Voltage AC Control of SMA Wires," in ASME 2020 Conference on Smart Materials, Adaptive Structures and Intelligent Systems, SMASIS 2020, 2020, p. V001T02A003, doi: https://doi.org/10.1115/SMASIS2020-2238.

[18] P. Motzki and S. Seelecke, "Bi-stable SMA Actuator," in Actuator 16 - 15th International Conference on New Actuators, 2016, pp. 317-320, doi: 10.13140/RG.2.2.12065.20325.

[19] P. Motzki and S. Seelecke, "Bistable Actuator Device Having A Shape Memory Element," US 2019/0203701 A1, 2019.

[20] F. Welsch, S.-M. Kirsch, P. Motzki, M. Schmidt, and S. Seelecke, “Vacuum Gripper System Based on Bis table SMA Actuation," in ASME 2018 Conference on Smart Materials, Adaptive Structures and Intelligent Systems, 2018, no. 51944, p. V001T04A014, doi: 10.1115/SMASIS2018-7980.

[21] S.-M. Kirsch, F. Welsch, M. Schmidt, P. Motzki, and S. Seelecke, “Bistable SMA Vacuum Suction Cup," in Actuator 18 - 16th International Conference on New Actuators, 2018, pp. 1-4.

[22] P. Motzki, T. Gorges, M. Kappel, M. Schmidt, G. Rizzello, and S. Seelecke, “High-Speed and HighEfficiency Shape Memory Alloy Actuation," Smart Mater. Struct., vol. 27, no. 7, p. 075047, 2018, doi: 
10.1088/1361-665X/aac9e1.

(c) (1)

BY
(C) 2020 by the authors; licensee MDPI, Basel, Switzerland. This article is an open access article dis tribute $\mathrm{d}$ under the terms and conditions of the Creative Commons by Attribution (CC-BY) license (http://creativecommons.org/licenses/by/4.0/). 